\title{
Control Study of Short-Term Aftereffect in Diving Exposure Related Pulmonary Ventilation Function
}

\section{Hua CHENG}

Sport Science School, Lingnan Normal University, Zhanjiang City, Guangdong Province, China
${ }^{*}$ Corresponding authors

Hua CHENG, Sport Science School, Lingnan Normal University, Chikan District, Zhanjiang City, Guangdong Province, 524048, China

Submitted: 15 March2021;Accepted:22 March 2021;Published: 05 Apr 2021

Citation: Hua CHENG (2021) Control Study of Short-Term Aftereffect in Diving Exposure Related Pulmonary Ventilation Function. Medical \&amp; Clinical Research 6(4): 507-517.

\begin{abstract}
Objective: Ventilation attenuation often happened in professional divers due to long-term cumulative effects in diving exercises. By case-control experiments, we observed the immediate effects of pulmonary ventilation before and after the exposure of 12m-depth underwater for $20 \mathrm{~min}$ to discuss the relationships between the short-term and long-time effects caused by diving environment.
\end{abstract}

\begin{abstract}
Methods: Participants were randomly assigned into the Experimental Group (EG) who stayed for 20 min under 12-m water environment or the Control Group (CG) who stayed in hyperbaric chamber under the pressure of 2.2ATA. Pulmonary ventilation function parameters including $V C, F V C, M V V$ and $M V$ were detected respectively before and after hyperbaric exposure by the Spirometer. Immediate effects of pulmonary ventilation before and after diving were compared by paired $t$ test to reveal the different influence caused by environmental pressure.
\end{abstract}

Results: The value of VC appears to rise while the MV, MVV were detected decreased after the exposure of 2.2ATA of environment pressure for $20 \mathrm{~min}$ in the two groups. VC increased more significantly in the $C G(t=-1.26, p=0.23)$ after hyperbaric exposure, that leads to the FEV1.0\%t ( $=F E V 1.0 / V C \%)$ increase in $E G(t=-0.73, p=0.48)$ while decrease in $C G$ $(t=0.42, p=0.17)$. The same trend in $V C, M V$ and $M V V$ after high pressure exposure in the two groups mainly due to the effect of common factors - the pressure. In addition to the impact of the pressure itself, EG members also face diving related immersion effect, influence of diving equipment load and water under low temperature. The FVC is detected decreased in $E G(t=1.21, P=0.25)$ while it increased in $C G(t=-0.42, P=0.68)$, but the differences are not significant and couldn't affect the measured FEV1.0\% ( $=F E V 1.0 / F V C \%)$ values showed both increasing in $E G(t=-1.48, P=0.16)$ and in $C G(t=-0.23, P$ $=0.82$ ). The expiratory flow rate including PEF, FEF25-75, MEF75, MEF50 increased in EG (t are -0.72, $-0.69,-0.87$ and -0.36 respectively with $P$ all greater than 0.05 ) while decreased in $C G$ (t are $1.67,0.50,1.53$ and 0.71 respectively with $P$ all greater than 0.05). MEF25 is the expiratory flow index of not affected by respiratory muscle force and the measured values of MEF25 increased in the $E G(t=-0.68, P=0.51)$ and in the $C G(t=-0.36, P=0.72)$.

Conclusion: Water immersion and water temperature during diving exposure could cause and accelerate hemodynamic changes in pulmonary circulation induced pulmonary interstitial edema and led to the increase of external respiratory work. Instant effects of diving exposure in the study are quite consistent with the long-term cumulative effect of professional divers in previous research, which is FVC reduced because small airway become narrower. The results illustrate even the small depth of short-range diving exercise have definite influences on pulmonary ventilation, which mainly comes from the environmental factor but not the pressure increases.

Keywords: Pulmonary Ventilation, Diving Exposure, Immersion Effect, Hyperbaric Exposure, SCUBA Diving.

\section{Abbreviation}

Tidal Volume: TV

Inspiratory Reserve Volume: IRV

Expiratory Reserve Volume: ERV
Residual Volume: RV

Inspiratory Capacity: IC

Vital Capacity: VC

Function Residual Capacity: FRC 
Total Lung Capacity: TLC

Minute Ventilation: MV

Maximal Voluntary Ventilation: MVV

Forced Vital Capacity: FVC

Forced Expiratory Volume in one second: $\mathrm{FEV}_{1.0}$

Ratio of $\mathrm{FEV}_{1}$ to $\mathrm{FVC}$ : $\mathrm{FEV}_{1.0} / \mathrm{FVC}\left(\mathrm{FEV}_{1.0} \%\right)$

Ratio of $\mathrm{FEV}_{1}^{1}$ to VC: $\mathrm{FEV}_{1.0}{ }^{1.0} \mathrm{VC}\left(\mathrm{FEV}_{1.0} \% \mathrm{ot}\right)$

Forced Expiratory Flow: $\mathrm{FEF}_{25 \sim 75} \%$

Peak Expiratory Flow: PEF

Forced Expiratory Flow after 25\% of the FVC has been exhaled: $\mathrm{FEF}_{25} \%\left(\mathrm{MEF}_{75}\right)$

Forced Expiratory Flow after $50 \%$ of the FVC has been exhaled: $\mathrm{FEF}_{50} \%\left(\mathrm{MEF}_{50}\right)$

Forced Expiratory Flow after $75 \%$ of the FVC has been exhaled: $\mathrm{FEF}_{75}\left(\mathrm{MEF}_{25}\right)$

Ventilation Reserve\%: VR\%

Respiratory Rate: RR

\section{Interview}

Though confront with some medical risk factors, SCUBA Diving still is a popular sport [1]. SCUBA Diving requires inhalation of compressed air through the breathing tube, and the pressure of the breath is exacerbated by strikingly inhomogeneous inhalation patterns, which makes the lung organ become one of the most vulnerable organs [2]. Under pressure exposed, inhalation of high density gas breathing can lead to increasing work of breathing. Oxygen partial pressure and the oxygen toxicity effect of respiratory membrane and micro bubbles induced inflammation in pulmonary circulation during the decompression process [3-5]. The effects of functional hyperinflation or bronchial obstruction lead to lung ventilation impairment of the obstructive type. In addition to the causes of high pressure exposure, sports produce capillary leakage underwater and immersion in water increase stress on pulmonary capillaries and result in hemodynamic pulmonary edema $[6,7]$. When diving, hypothermia, hyperopia, hydrostatic pressure increase and strenuous exercise all induced pulmonary circulation change rapidly promote the occurrence of pulmonary edema further affected the lung ventilation function [8]. The FVC is significantly reduced by about $240 \mathrm{ml} / 3-5$ yrs and $400 \mathrm{ml} / 5-9$ yrs according to previous physical examination of professional divers [9]. Exposure to diving affects the small airways, a tendency to reduce bronchial congestion, extend to bronchiectasis, and may lead to changes in lung function $[10,11]$. Prevalence indicated that 6 to 15 percent of professional divers have a tendency to of airflow obstruction as the diving experience grows. Airways narrowing might be due to diving-induced loss of lung elastic tissue $[12,13]$. It causes the reduction of $\mathrm{FEV}_{1.0}$. In the meantime diving exposure affects the vital capacity and the forced expiratory flow rate [14]. Although the cumulative effect of lung function in professional divers has been observed before, but the relationship of all possible influence factors not been clearly explained $[15,16]$. This study observed changes of pulmonary ventilation function in diving experiment and hyperbaric chamber pressure exposure from a single no-decompression, small-depth dive. Diving experimental environment pressure settings are referenced to the safety standards of decompression procedures through controlling diving depth and time of hyperbaric exposure, compression and decompression speed [17]. In this paper, the relationship between the immediate effect of pulmonary ventilation and the cumulative effect of pulmonary function was discussed by comparing the immediate effects of pulmonary ventilation before and after the same pressure change.

\section{Participants and Methods \\ Participants}

Healthy volunteers are enrolled as participants in the experiment, whose maximum diving depth no less than the maximum depth of $20 \mathrm{~m}$ underwater and the maximum duration of staying at the same depth for no less than $5 \mathrm{~min}$.Participants who have been affected by acute respiratory diseases or other diseases could affect the normal conditions of diving and those who had suffered from diving disease before should be ruled out. Informed consent forms were issued and signed to ensure all participants were informed of the detailed of the research procedure and allow exiting the experiment at any time.

\section{Methods}

Tools for Measurement Lung function measuring device (MINATO AS -505) is a widely used for easy operation, method standardization, and high precision spirometer. The main measurement indicators are $\mathrm{FEV}_{1.0}, \mathrm{FVC}$, and $\mathrm{FEV}_{1.0} \%$ (that is,

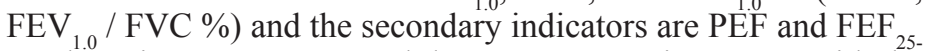
75. The spirometer can record the current-capacity curve. With the $0-14 \mathrm{~L} / \mathrm{S}$ in flow range, $\pm 3 \%$ or $\pm 0.01 \mathrm{~L} / \mathrm{S}$ of measuring range in accuracy, $10 \mathrm{~L}$ in maximum capacity, $\pm 3 \%$ or $50 \mathrm{~mL}$ in capacity accuracy, the spirometer is repeatable, responsive and reliable for ventilation measurement [18]. For the participants, cola, coffee, strong tea is forbidden on the day of inspection. Before testing, participants are suggested to not eating too much in $2 \mathrm{hrs}$, prohibited smoking in 1hour and not allowed strenuous exercise in $30 \mathrm{~min}$. After containing the blowtorch with the mouth steadily, worn nose clips properly, breathing in and out the indoor air calmly for 20 30sec, and wait for the baseline of the tidal breathing to plateau at least four breathing cycles, then the measure start.

Determine the avoidable measurements values should be reference to at least two times of measurements. Rest at least $1 \mathrm{~min}$ in the middle of each measurement. The error between the best and the suboptimal values should be under than $0.15 \mathrm{~L}$. The normal range of lung volume indicators is a $95 \%$ confidence interval for the expected value equation. Those below the lower limit of normal (LLN) and the upper limit of normal range (ULN) are considered abnormal. The variation in the volume of lung capacity is distinct. The normal range of the volume of lung capacity is simplified to the percentage of the expected value, and the normal range is generally expected to be $\pm 20 \%$.

Basic physiological parameters and respiratory function were tested two days before the experiment, which include height, weight, and the Body Mass Index (BMI), such as follows:

$$
B M I=W e i g h t(\mathrm{Kg}) \div \operatorname{Height}(\mathrm{cm})^{2}
$$

Ventilation functions' testing is strictly according to the instructions of the spirometer. First enter the participants' gender, age, height, weight, and adjust the prediction model into Baldwin mode. Ventilation values' expected regression equation are as follows: 


$$
\begin{aligned}
& V C_{\text {male }}(\mathrm{mL})=\{27.63-[0.112 \times \operatorname{Age}(\mathrm{y})]\} \times \operatorname{Height}(\mathrm{cm}) \\
& V C_{\text {female }}(\mathrm{mL})=\{21.78-[0.101 \times \operatorname{Age}(\mathrm{y})]\} \times \operatorname{Height}(\mathrm{cm}) \\
& M V V_{\text {male }}(\mathrm{L})=\{86.4-[0.522 \times \operatorname{Age}(\mathrm{y})]\} \times B S A\left(\mathrm{~m}^{2}\right) \\
& M V V_{\text {female }}(\mathrm{L})=\{71.3-[0.474 \times \operatorname{Age}(\mathrm{y})]\} \times B S A\left(\mathrm{~m}^{2}\right)
\end{aligned}
$$

Each participant will repeat testing the values of $\mathrm{VC}, \mathrm{FVC}, \mathrm{MV}$, and MVV for more than two times. Testing not only arrive at the figures of VC, FVC, FEV ${ }_{1.0}, \mathrm{FEV} 1.0 \%, \mathrm{PEF}, \mathrm{FEF}_{25-75}, \mathrm{MEF}_{50}$, $\mathrm{MEF}_{25}, \mathrm{MVV}$ in measured values and predicted values, but also the figures of TV, IRV, ERV, IC and MV in measured values are concluded.

$$
\begin{aligned}
& V C(\mathrm{~L})=\boldsymbol{T V}(\mathrm{L})+\boldsymbol{I R V}(\mathrm{L})+\boldsymbol{E R V}(\mathrm{L}) \\
& \boldsymbol{I C}(\mathrm{L})=\boldsymbol{T V}(\mathrm{L})+\boldsymbol{I R V}(\mathrm{L}) \\
& V_{1.0} \%=\frac{F E V_{1.0}(\mathrm{~L})}{F V C(\mathrm{~L})} \times 10 \\
& F E V_{1.0} \% t=\frac{F E V_{1.0}(\mathrm{~L})}{V C(\mathrm{~L})} \times 100 \% \\
& M V(\mathrm{~L})=\boldsymbol{T V}(\mathrm{L}) \times \boldsymbol{R} R(\mathrm{bpm}) \\
& V R \%=\frac{M V V(\mathrm{~L})-M V(\mathrm{~L})}{M V V(\mathrm{~L})} \times 100 \%
\end{aligned}
$$

\section{Experiments Settings}

The participants were classified into the SCUBA Diving group (the experimental group, EG) and the hyperbaric chamber group (the control group, CG) according to the match of their indicators of the Age, Gender, BMI and FVC.

Participants in the experimental group wore tight wet diving suits and carried scuba tank of 12L. Each of them made a dive to $12 \mathrm{~m}$-depth under water from the surface at $6 \mathrm{~m} / \mathrm{min}$ and stops at $12 \mathrm{~m}$ for $20 \mathrm{~min}$ and then ascends to the surface at the same speed. The parameters values of ventilations (VC, FVC, MV and MVV) are assessed by the instructor right after surfacing.

According to Boyle-Mariotte law, the actual ventilation per minute underwater can be calculated as the following procedure.

$$
\begin{aligned}
& P_{1}(\mathrm{kPa}) V_{1}(\mathrm{~L})=P_{2}(\mathrm{kPa}) V_{2}(\mathrm{~L}) \text { (or) } P(\mathrm{kPa}) V(\mathrm{~L})=k \\
& \because \quad \mathrm{T}_{\text {underwater }}(\min )=T_{\text {down }}(\min )+T_{\text {stay }}(\min )+T_{\text {up }}(\text { min }) \\
& V_{2}(L)=\frac{V_{1}(L) \times P_{1}(k P a)}{P_{2}(k P a)}=\frac{V_{1}(L) \times \Delta P(k P a)}{P_{2}(k P a)} \\
& \mathrm{MV}_{\text {underwater }}=\mathrm{TV} \times \mathrm{RR}=V_{2}(L) \div T_{\text {underwater }}(\mathrm{min}) \\
& \therefore \quad \mathbf{M V}_{\text {underwater }}=\frac{\mathbf{V}_{1}(L) \times \Delta \mathbf{P}(k P a) / \mathbf{P}_{2}(k P a)}{T_{\text {down }}(\min )+T_{\text {stay }}(\min )+T_{\text {up }}(\min )}
\end{aligned}
$$

\section{Change of Minute Ventilation underwater in EG}

The mean measured values of MV in the whole EG before experimental exposure was $30.09 \pm 14.27 \mathrm{~L}$. In the single dive bottom time after submersion, calculations according to the equation (12), the divers' average MV were down to 24.01 \pm 5.04 $L$. The average measured value of MV right after emerging from the water is up to $27.98 \pm 12$. $99 \mathrm{~L}$. The fluctuation of MV measured values are shown in the following Table $1 \&$ Figure 1. 
Table 1: Minute Ventilation (MV) of Participants in EG Pre- and Post- Hyperbaric Exposure

\begin{tabular}{|c|c|c|c|c|c|c|c|c|}
\hline \multirow{2}{*}{$\begin{array}{l}\text { Gender } \\
(\text { male }=1, \\
\text { female }=2)\end{array}$} & \multirow{2}{*}{$\begin{array}{c}\text { Age } \\
(y)\end{array}$} & \multirow{2}{*}{$\begin{array}{c}\text { Weight } \\
\text { (Kg) }\end{array}$} & \multirow{2}{*}{$\begin{array}{l}\text { Height } \\
\text { (cm) }\end{array}$} & \multirow[t]{2}{*}{ BMI } & \multicolumn{4}{|c|}{ MV(L) } \\
\hline & & & & & $\operatorname{Pre}^{-1}$ & Underwater $^{2}$ & Post $^{-3}$ & $\bar{X} \pm \mathrm{SD}$ \\
\hline 1 & 23 & 63 & 175.00 & 20.57 & 35.68 & 25.53 & 34.92 & $32.04 \pm 5.65$ \\
\hline 1 & 21 & 67 & 170.00 & 23.18 & 14.18 & 35.74 & 14.34 & $21.42 \pm 12.40$ \\
\hline 1 & 22 & 78 & 172.00 & 26.37 & 6.18 & 22.98 & 7.14 & $12.10 \pm 9.43$ \\
\hline 1 & 22 & 68 & 175.00 & 22.20 & 44.85 & 30.64 & 38.64 & $38.04 \pm 7.12$ \\
\hline 1 & 20 & 65 & 173.00 & 21.72 & 41.82 & 25.53 & 40.72 & $36.02 \pm 9.10$ \\
\hline 1 & 21 & 69 & 179.00 & 21.53 & 26.06 & 19.20 & 12.65 & $19.30 \pm 6.71$ \\
\hline 1 & 21 & 58 & 170.00 & 20.07 & 34.24 & 28.8 & 33.38 & $32.14 \pm 2.92$ \\
\hline 2 & 23 & 53 & 166.00 & 19.23 & 30.49 & 24.00 & 33.01 & $29.17 \pm 4.65$ \\
\hline 1 & 21 & 67 & 179.00 & 20.91 & 46.56 & 20.17 & 39.82 & $35.52 \pm 13.71$ \\
\hline 1 & 21 & 67.2 & 176.00 & 21.69 & 14.52 & 20.17 & 20.34 & $18.34 \pm 3.31$ \\
\hline 1 & 23 & 70 & 179.00 & 21.85 & 43.19 & 20.17 & 41.08 & $34.81 \pm 12.73$ \\
\hline 1 & 22 & 60 & 165.00 & 22.04 & 49.27 & 17.80 & 44.60 & $37.22 \pm 16.98$ \\
\hline 1 & 23 & 62 & 171.00 & 21.20 & 19.58 & 20.34 & 17.04 & $18.99 \pm 1.73$ \\
\hline 2 & 21 & 53.7 & 165.00 & 19.72 & 14.68 & 25.00 & 14.07 & $17.92 \pm 6.14$ \\
\hline $\bar{X} \pm \mathrm{SD}$ & $21.71 \pm 0.99$ & $64.35 \pm 6.71$ & $172.50 \pm 4.97$ & $21.59 \pm 1.73$ & $30.09 \pm 14.27$ & $24.01 \pm 5.04$ & $27.98 \pm 12.99$ & $27.36 \pm 11.51$ \\
\hline
\end{tabular}

Pre $^{-1}$ :pre-hyperbaric exposure; Underwater ${ }^{2}$; underwater hyperbaric exposure; Post ${ }^{-3}$; Post-hyperbaric exposure

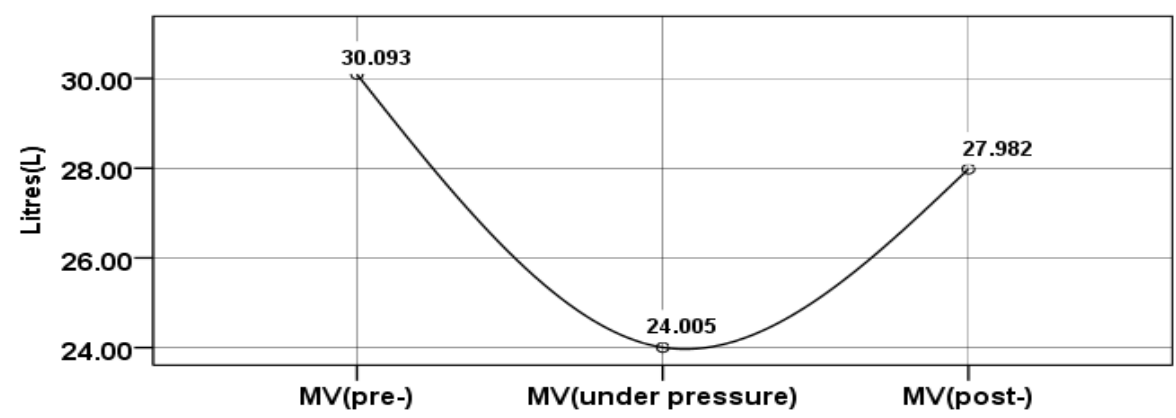

Figure 1: The variation of ventilation in different experimental stages pre-: pre-hyperbaric exposure; underwater: underwater hyperbaric exposure; post-: post-hyperbaric exposure; MV: Minute ventilation (L)

Changes on measured values of the pulmonary ventilation in two groups pre- and post- hyperbaric exposure

Paired sample $\mathrm{t}$ tests compare the measured values of ventilation parameters in EG (Table 2) \& CG (Table 3) pre- and post- hyperbaric exposure. The same $t$ test analysis also performed to the values of the measured/predicted \% of the pulmonary ventilation in order to reduce the errors, which confirm the validity of the variation of the measured value of pulmonary ventilation. 
Table 2: Measured Values of the Pulmonary Ventilation in EG pre- and post- Hyperbaric Exposure

\begin{tabular}{|c|c|c|c|c|c|c|c|c|c|c|c|c|c|}
\hline & \multicolumn{11}{|c|}{ Pairwise difference } & \multirow{3}{*}{ df } & \multirow{3}{*}{ Sig } \\
\hline & \multirow[t]{2}{*}{ Measured Value } & \multirow[t]{2}{*}{$X$} & \multirow[t]{2}{*}{$\mathbf{N}$} & \multirow[t]{2}{*}{ SD } & \multirow[t]{2}{*}{ SE } & \multirow[t]{2}{*}{$\bar{X}$} & \multirow[t]{2}{*}{ SD } & \multirow[t]{2}{*}{ SE } & \multicolumn{2}{|c|}{$95 \%$} & \multirow[t]{2}{*}{$\mathbf{t}$} & & \\
\hline & & & & & & & & & $\begin{array}{l}\text { Upper } \\
\text { limit }\end{array}$ & $\begin{array}{c}\text { Lower } \\
\text { limit }\end{array}$ & & & \\
\hline \multirow[t]{2}{*}{1} & VC(pre-) & 3.61 & 14 & 0.56 & 0.15 & \multirow[t]{2}{*}{-0.06} & \multirow[t]{2}{*}{0.16} & \multirow[t]{2}{*}{0.04} & \multirow[t]{2}{*}{-0.15} & \multirow[t]{2}{*}{0.04} & \multirow[t]{2}{*}{-1.26} & \multirow[t]{2}{*}{13} & 0.23 \\
\hline & $\mathrm{VC}($ post-) & 3.67 & 14 & 0.48 & 0.13 & & & & & & & & \\
\hline 2 & TV(pre-) & 1.50 & 14 & 0.72 & 0.19 & -0.07 & 0.40 & 0.11 & -0.30 & 0.16 & -0.67 & 13 & 0.52 \\
\hline & TV(post-) & 1.57 & 14 & 0.71 & 0.19 & & & & & & & & \\
\hline 3 & IRV(pre-) & 0.99 & 14 & 0.45 & 0.12 & -0.06 & 0.26 & 0.07 & -0.21 & 0.09 & -0.81 & 13 & 0.43 \\
\hline & IRV(post-) & 1.05 & 14 & 0.47 & 0.13 & & & & & & & & \\
\hline 4 & ERV(pre-) & 1.12 & 14 & 0.36 & 0.10 & 0.07 & 0.35 & 0.09 & -0.13 & 0.27 & 0.76 & 13 & 0.46 \\
\hline & ERV(post-) & 1.05 & 14 & 0.35 & 0.09 & & & & & & & & \\
\hline 5 & IC(pre-) & 2.49 & 14 & 0.59 & 0.16 & -0.13 & 0.40 & 0.11 & -0.36 & 0.11 & -1.17 & 13 & 0.26 \\
\hline & IC(post-) & 2.62 & 14 & 0.49 & 0.13 & & & & & & & & \\
\hline 6 & FVC(pre-) & 3.64 & 14 & 0.57 & 0.15 & 0.09 & 0.28 & 0.07 & -0.07 & 0.25 & 1.21 & 13 & 0.25 \\
\hline & FVC(post-) & 3.55 & 14 & 0.54 & 0.14 & & & & & & & & \\
\hline 7 & FEV1.0(pre-) & 2.68 & 14 & 0.94 & 0.25 & -0.16 & 0.83 & 0.22 & -0.64 & 0.32 & -0.73 & 13 & 0.48 \\
\hline & FEV1.0(post-) & 2.84 & 14 & 0.59 & 0.16 & & & & & & & & \\
\hline 8 & FEV1.0\%(pre-) & $72.99 \%$ & 14 & $21.25 \%$ & $5.68 \%$ & $-7.94 \%$ & $20.14 \%$ & $5.38 \%$ & $-19.57 \%$ & $3.68 \%$ & -1.48 & 13 & 0.16 \\
\hline & FEV1.0\%(post-) & $80.94 \%$ & 14 & $15.63 \%$ & $4.18 \%$ & & & & & & & & \\
\hline 9 & $\begin{array}{l}\text { FEV1.0/ } \\
\text { VC\%(pre-) }\end{array}$ & $73.68 \%$ & 14 & $22.32 \%$ & $5.97 \%$ & $-4.05 \%$ & $20.92 \%$ & $5.59 \%$ & $-16.13 \%$ & $8.02 \%$ & -0.73 & 13 & 0.48 \\
\hline & $\begin{array}{l}\text { FEV1.0/VC\% } \\
\text { (post-) }\end{array}$ & $77.73 \%$ & 14 & $14.66 \%$ & $3.92 \%$ & & & & & & & & \\
\hline 10 & PEF(pre-) & 3.97 & 14 & 2.01 & 0.54 & -0.31 & 1.60 & 0.43 & -1.23 & 0.62 & -0.72 & 13 & 0.49 \\
\hline & PEF(post-) & 4.28 & 14 & 1.86 & 0.50 & & & & & & & & \\
\hline 11 & FEF25-75(pre-) & 2.92 & 14 & 1.54 & 0.41 & -0.25 & 1.34 & 0.36 & -1.02 & 0.52 & -0.69 & 13 & 0.50 \\
\hline & FEF25-75(post-) & 3.17 & 14 & 1.31 & 0.35 & & & & & & & & \\
\hline 12 & MEF75(pre-) & 3.68 & 14 & 1.98 & 0.53 & -0.37 & 1.58 & 0.42 & -1.28 & 0.55 & -0.87 & 13 & 0.40 \\
\hline & MEF75(post-) & 4.05 & 14 & 1.86 & 0.50 & & & & & & & & \\
\hline 13 & MEF50(pre-) & 3.14 & 14 & 1.70 & 0.45 & -0.13 & 1.30 & 0.35 & -0.88 & 0.63 & -0.36 & 13 & 0.72 \\
\hline & MEF50(post-) & 3.26 & 14 & 1.23 & 0.33 & & & & & & & & \\
\hline 14 & MEF25(pre-) & 2.01 & 14 & 0.99 & 0.26 & -0.13 & 0.71 & 0.19 & -0.54 & 0.28 & -0.68 & 13 & 0.51 \\
\hline & MEF25(post-) & 2.14 & 14 & 0.88 & 0.24 & & & & & & & & \\
\hline 15 & MVV(pre-) & 60.05 & 14 & 22.90 & 6.12 & 1.09 & 17.69 & 4.73 & -9.12 & 11.31 & 0.23 & 13 & 0.82 \\
\hline & MVV(post-) & 58.96 & 14 & 14.34 & 3.83 & & & & & & & & \\
\hline 16 & MV(pre-) & 30.09 & 14 & 14.27 & 3.81 & 2.11 & 4.63 & 1.24 & -0.56 & 4.78 & 1.71 & 13 & 0.11 \\
\hline & MV(post-) & 27.98 & 14 & 12.99 & 3.47 & & & & & & & & \\
\hline 17 & RR(pre-) & 21.50 & 14 & 8.21 & 2.20 & 2.12 & 6.02 & 1.61 & -1.36 & 5.59 & 1.32 & 13 & 0.21 \\
\hline & RR(post-) & 19.39 & 14 & 8.07 & 2.16 & & & & & & & & \\
\hline 18 & VR\%(pre-) & $49.20 \%$ & 14 & $18.27 \%$ & $4.88 \%$ & $-3.03 \%$ & $13.39 \%$ & $3.58 \%$ & $-10.76 \%$ & $4.70 \%$ & -0.85 & 13 & 0.41 \\
\hline & VR\%(post-) & $52.23 \%$ & 14 & $19.14 \%$ & $5.12 \%$ & & & & & & & & \\
\hline
\end{tabular}

pre-: pre-hyperbaric exposure; post-: post-hyperbaric exposure;

$* \mathrm{P}$-value $<0.05$, Difference was statistically significant; **P-value $<0.01$, Difference was significant statistical significance 
Table 3: Measured Values of the Pulmonary Ventilation in CG pre- and post- Hyperbaric Exposure

\begin{tabular}{|c|c|c|c|c|c|c|c|c|c|c|c|c|c|}
\hline \multirow{2}{*}{\multicolumn{3}{|c|}{ CG: Control Group(N=20) }} & \multicolumn{11}{|c|}{ Paired Samples Test } \\
\hline & & & \multicolumn{11}{|c|}{ Pairwise difference } \\
\hline & \multirow[t]{2}{*}{ Measured Value } & \multirow[t]{2}{*}{$\overline{\bar{X}}$} & \multirow[t]{2}{*}{$\mathbf{N}$} & \multirow[t]{2}{*}{ SD } & \multirow[t]{2}{*}{ SE } & \multirow[t]{2}{*}{$\bar{X}$} & \multirow[t]{2}{*}{ SD } & \multirow[t]{2}{*}{ SE } & \multicolumn{2}{|l|}{$95 \%$ cl } & \multirow[t]{2}{*}{$\mathbf{t}$} & \multirow[t]{2}{*}{ df } & \multirow[t]{2}{*}{ Sig } \\
\hline & & & & & & & & & \begin{tabular}{|l|} 
Upper \\
limit
\end{tabular} & \begin{tabular}{|l|}
$\begin{array}{l}\text { Lower } \\
\text { limit }\end{array}$ \\
\end{tabular} & & & \\
\hline \multirow[t]{2}{*}{1} & VC(pre-) & 3.56 & 20 & 0.48 & 0.11 & \multirow[t]{2}{*}{-0.13} & \multirow[t]{2}{*}{0.20} & \multirow[t]{2}{*}{0.05} & \multirow[t]{2}{*}{-0.23} & \multirow[t]{2}{*}{-0.04} & \multirow[t]{2}{*}{-2.92} & 19 & $0.009 * *$ \\
\hline & $\mathrm{VC}$ (post-) & 3.69 & 20 & 0.51 & 0.11 & & & & & & & & \\
\hline 2 & TV(pre-) & 1.41 & 20 & 0.56 & 0.13 & -0.06 & 0.42 & 0.09 & -0.26 & 0.13 & -0.66 & 19 & 0.52 \\
\hline & TV(post-) & 1.47 & 20 & 0.68 & 0.15 & & & & & & & & \\
\hline 3 & IRV(pre-) & 0.89 & 20 & 0.33 & 0.07 & -0.27 & 0.34 & 0.08 & -0.43 & -0.11 & -3.48 & 19 & $0.003 * *$ \\
\hline & IRV(post-) & 1.16 & 20 & 0.47 & 0.11 & & & & & & & & \\
\hline 4 & ERV(pre-) & 1.25 & 20 & 0.38 & 0.08 & 0.18 & 0.25 & 0.06 & 0.07 & 0.30 & 3.34 & 19 & $0.003 * *$ \\
\hline & ERV(post-) & 1.07 & 20 & 0.37 & 0.08 & & & & & & & & \\
\hline 5 & IC(pre-) & 2.30 & 20 & 0.38 & 0.08 & -0.33 & 0.26 & 0.06 & -0.45 & -0.21 & -5.68 & 19 & $0.000 * *$ \\
\hline & IC(post-) & 2.62 & 20 & 0.50 & 0.11 & & & & & & & & \\
\hline 6 & FVC(pre-) & 3.68 & 20 & 0.46 & 0.10 & -0.02 & 0.20 & 0.04 & -0.11 & 0.07 & -0.42 & 19 & 0.68 \\
\hline & FVC(post-) & 3.70 & 20 & 0.56 & 0.12 & & & & & & & & \\
\hline 7 & FEV1.0(pre-) & 3.09 & 20 & 0.53 & 0.12 & -0.02 & 0.39 & 0.09 & -0.20 & 0.16 & -0.24 & 19 & 0.82 \\
\hline & FEV1.0(post-) & 3.11 & 20 & 0.52 & 0.12 & & & & & & & & \\
\hline 8 & FEV1.0\%(pre-) & $84.21 \%$ & 20 & $12.22 \%$ & $2.73 \%$ & \begin{tabular}{|l|}
$-0.46 \%$ \\
\end{tabular} & $8.89 \%$ & $1.99 \%$ & $-4.62 \%$ & $3.71 \%$ & -0.23 & 19 & 0.82 \\
\hline & FEV1.0\%(post-) & $84.66 \%$ & 20 & $10.81 \%$ & $2.42 \%$ & & & & & & & & \\
\hline 9 & $\begin{array}{l}\text { FEV1.0/ } \\
\text { VC\%(pre-) }\end{array}$ & $87.65 \%$ & 20 & $12.29 \%$ & $2.75 \%$ & $2.97 \%$ & $9.37 \%$ & $2.10 \%$ & $-1.42 \%$ & $7.35 \%$ & 1.42 & 19 & 0.17 \\
\hline & $\begin{array}{l}\text { FEV1.0/VC\% } \\
\text { (post-) }\end{array}$ & $84.68 \%$ & 20 & $10.02 \%$ & $2.24 \%$ & & & & & & & & \\
\hline 10 & PEF(pre-) & 4.72 & 20 & 1.37 & 0.31 & 0.41 & 1.11 & 0.25 & -0.11 & 0.93 & 1.67 & 19 & 0.11 \\
\hline & PEF(post-) & 4.31 & 20 & 1.18 & 0.26 & & & & & & & & \\
\hline 11 & FEF25-75(pre-) & 3.46 & 20 & 0.94 & 0.21 & 0.09 & 0.84 & 0.19 & -0.30 & 0.49 & 0.50 & 19 & 0.62 \\
\hline & FEF25-75(post-) & 3.37 & 20 & 0.97 & 0.22 & & & & & & & & \\
\hline 12 & MEF75(pre-) & 4.49 & 20 & 1.37 & 0.31 & 0.37 & 1.08 & 0.24 & -0.13 & 0.87 & 1.53 & 19 & 0.14 \\
\hline & \begin{tabular}{|l|} 
MEF75(post-) \\
\end{tabular} & 4.12 & 20 & 1.23 & 0.28 & & & & & & & & \\
\hline 13 & MEF50(pre-) & 3.74 & 20 & 1.01 & 0.23 & 0.13 & 0.85 & 0.19 & -0.26 & 0.53 & 0.71 & 19 & 0.49 \\
\hline & MEF50(post-) & 3.60 & 20 & 1.00 & 0.22 & & & & & & & & \\
\hline 14 & MEF25(pre-) & 2.43 & 20 & 0.62 & 0.14 & -0.06 & 0.70 & 0.16 & -0.38 & 0.27 & -0.36 & 19 & 0.72 \\
\hline & MEF25(post-) & 2.48 & 20 & 0.78 & 0.17 & & & & & & & & \\
\hline 15 & MVV(pre-) & 56.56 & 20 & 17.47 & 3.91 & 1.36 & 10.28 & 2.30 & -3.45 & 6.17 & 0.59 & 19 & 0.56 \\
\hline & MVV(post-) & 55.20 & 20 & 15.62 & 3.49 & & & & & & & & \\
\hline 16 & MV(pre-) & 27.27 & 20 & 10.52 & 2.35 & 1.11 & 5.08 & 1.14 & -1.26 & 3.49 & 0.98 & 19 & 0.34 \\
\hline & MV(post-) & 26.15 & 20 & 9.89 & 2.21 & & & & & & & & \\
\hline 17 & RR(pre-) & 20.43 & 20 & 7.57 & 1.69 & 0.14 & 8.79 & 1.96 & -3.97 & 4.25 & 0.07 & 19 & 0.94 \\
\hline & RR(post-) & 20.29 & 20 & 10.13 & 2.27 & & & & & & & & \\
\hline 18 & VR\%(pre-) & $50.98 \%$ & 20 & $14.55 \%$ & $3.25 \%$ & $-1.53 \%$ & $8.99 \%$ & $2.01 \%$ & $-5.74 \%$ & $2.68 \%$ & -0.76 & 19 & 0.46 \\
\hline & VR\%(post-) & $52.51 \%$ & 20 & $12.52 \%$ & $2.80 \%$ & & & & & & & & \\
\hline
\end{tabular}


pre-: pre-hyperbaric exposure; post-: post-hyperbaric exposure;

$* P$-value $<0.05$, Difference was statistically significant; $* * P$-value $<0.01$, Difference was significant statistical significance
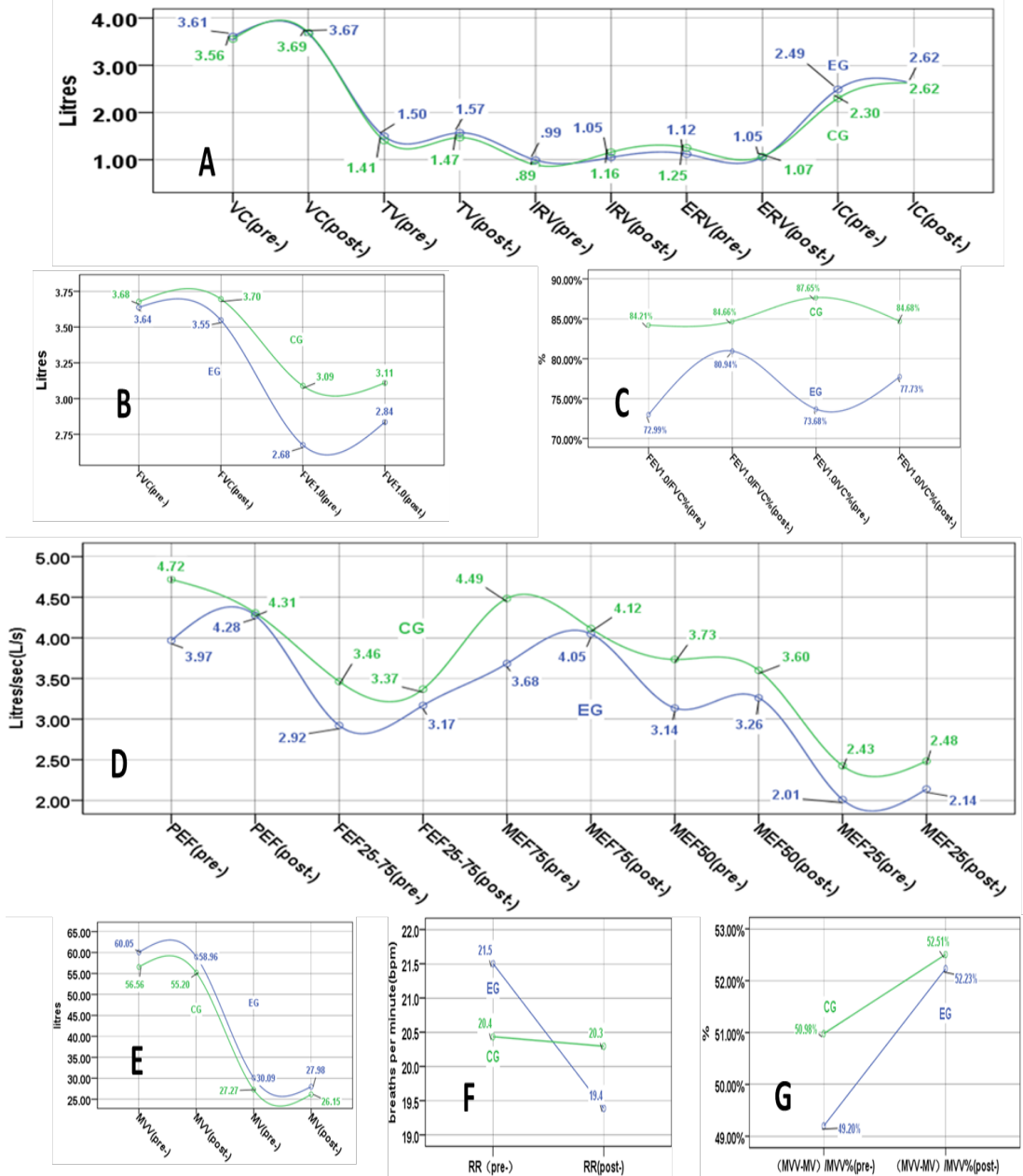

Figure 2: Comparison of Average in Measured Values of Ventilation Parameters in Both Groups Pre- and Post- Hyperbaric Exposure EG: Experimental Group; CG: Control Group pre-: pre-hyperbaric exposure; post-: post-hyperbaric exposure;

(A)VC: Vital Capacity (L); TV: Tidal Volume (L); IRV: Inspiratory Reserve Volume (L); ERV: Expiratory Reserve Volume (L); IC: Inspiratory Capacity(L)

(B)FVC: Forced Vital Capacity(L); FEV 1.0 : Forced Expiratory Volume in one second (L);

(C)FEV ${ }_{1.0} \%$ : a ratio of $\mathrm{FEV}_{1}$ to $\mathrm{FVC} \mathrm{FEV}_{1.0} / \mathrm{VC}^{2}$ : ratio of $\mathrm{FEV}_{1}$ to $\mathrm{VC}$;

(D)PEF: Peak Expiratory Flow(L/s); $\mathrm{FEF}_{25-75}$ : Forced Expiratory Flow(L/s); $\mathrm{MEF}_{75}$ : forced expiratory flow after $25 \%$ of the $\mathrm{FVC}$ has been exhaled(L/s); $\mathrm{MEF}_{50}$ : forced expiratory flow after $50 \%$ of the $\mathrm{FVC}$ has been exhaled(L/s); $\mathrm{MEF}_{25}$ : forced expiratory flow after $75 \%$ of the FVC has been exhaled(L/s);

(E)MVV: Maximal Voluntary Ventilation(L); MV: Minute Ventilation(L);

(RR: Respiratory Rate(bpm);

(G)VR\%: Ventilation Reserve\%=(MVV-MV)/MVV\%

By comparison, there are same change trends (Table $4 \&$ Table 7) and opposite trends (Table $5 \&$ Table 6 ) are found in two groups. 
The TV and IRV are increased, while the ERV is decreased after high pressure exposure. And because the IC consists of TV and IRV, and the VC consists of TV, IRV and ERV, so that the IC and $\mathrm{VC}$ also increase after high voltage exposure. The decline in
ERV will not offset the rise in TV and IRV. These changes were even more evident in the CG, which only under the mere $2.2 A T A$ pressure (Table 4 \& Figure 2-A).

Table 4: Tendency of ventilation function (TV, IRV, ERV, IC, VC) in EG and CG after exposure to high pressure

\begin{tabular}{|c|c|c|l|l|l|l|l|l|l|c|}
\hline & \multicolumn{2}{l}{ TV } & \multicolumn{2}{l|}{ IRV } & \multicolumn{2}{l|}{ ERV } & \multicolumn{2}{l|}{ VC } \\
\hline & tendency & P-Value & tendency & P-Value & tendency & P-Value & tendency & $P$-Value & tendency & $P$-Value \\
\hline EG & $\uparrow$ & $>0.05$ & $\uparrow$ & $>0.05$ & $\downarrow$ & $>0.05$ & $\uparrow$ & $>0.05$ & $\uparrow$ & $>0.05$ \\
\hline CG & $\uparrow$ & $>0.05$ & $\uparrow \uparrow$ & $<0.05$ & $\downarrow \downarrow$ & $<0.05$ & $\uparrow \uparrow$ & $<0.05$ & $\uparrow \uparrow$ & $<0.05$ \\
\hline
\end{tabular}

$\mathrm{FEV}_{1.0}$ showed an increase in both groups, indicating that the Forced Expiratory Volume in the first second of the high-pressure exposure both increased in two groups. The FVC showed an opposite trend in the EG and CG after the hyperbaric exposure, which was the decline in the EG and increased in the CG. Although the tendencies of FVC were inconsistent, the proportion of $\mathrm{FEV}_{1.0}$ to $\mathrm{FVC}$ in both groups still increased, indicated that the rise in the $\mathrm{FEV}_{1.0}$ of the $\mathrm{CG}$ seem to exceed the $\mathrm{FVC}$. Both $\mathrm{FEV}_{1.0} \% \mathrm{t}$ declined due to the increase of VC in both groups (Table 5, Figure 2-B \& 2-C).

Table 5: Tendency of ventilation function (FVC, $\left.\mathrm{FEV}_{1.0}, \mathrm{FEV}_{1.0} \%, \mathrm{FEV}_{1.0} \% \mathrm{t}\right)$ in $\mathrm{EG}$ and CG after exposure to high pressure

\begin{tabular}{|l|l|l|l|l|l|l|l|r|}
\hline & \multicolumn{3}{|l|}{ FVC } & \multicolumn{2}{l|}{ FEV $_{1.0}$} & FEV $_{1.0} \%$ & \multicolumn{2}{l|}{ FEV $_{1.0} \% \mathbf{~}$} \\
\hline & tendency & P-Value & tendency & P-Value & tendency & P-Value & tendency & P-Value \\
\hline EG & $\downarrow$ & $>0.05$ & $\uparrow$ & $>0.05$ & $\uparrow$ & $>0.05$ & $\downarrow$ & $>0.05$ \\
\hline CG & $\uparrow$ & $>0.05$ & $\uparrow$ & $>0.05$ & $\uparrow$ & $>0.05$ & $\uparrow$ & $>0.05$ \\
\hline
\end{tabular}

The expiratory gas flow rate (PFE, $\mathrm{FEF}_{25-75}, \mathrm{MEF}_{75}, \mathrm{MEF}_{50}, \mathrm{MEF}_{25}$ ) were all shown to increase in EG. But the PFE, $\mathrm{FEF}_{25-75}, \mathrm{MEF}_{75}$, $\mathrm{MEF}_{50}$ decreased while only $\mathrm{MEF}_{25}$ increased in CG (Table $6 \&$ Figure 2-D).

Table 6: Tendency of ventilation function ( $\mathrm{PFE}, \mathrm{FEF}_{25-75}, \mathrm{MEF}_{75}, \mathrm{MEF}_{50}, \mathrm{MEF}_{25}$ ) in EG and CG after exposure to high pressure

\begin{tabular}{|c|c|c|c|c|c|c|c|c|c|c|}
\hline & \multicolumn{2}{|l|}{ PEF } & \multicolumn{2}{|l|}{ FEF $_{25-75}$} & \multicolumn{2}{|l|}{$\mathbf{M E F}_{75}$} & \multicolumn{2}{|l|}{$\mathbf{M E F}_{50}$} & \multicolumn{2}{|c|}{$\mathrm{MEF}_{25}$} \\
\hline & tendency & P-Value & tendency & P-Value & tendency & P-Value & tendency & P-Value & & P-Value \\
\hline EG & $\uparrow$ & $>0.05$ & $\uparrow$ & $>0.05$ & $\uparrow$ & $>0.05$ & $\uparrow$ & $>0.05$ & $\uparrow$ & $>0.05$ \\
\hline CG & $\downarrow$ & $x>0.05$ & $\downarrow$ & $>0.05$ & $\downarrow$ & $>0.05$ & $\downarrow$ & $>0.05$ & $\uparrow$ & $>0.05$ \\
\hline
\end{tabular}

The MV, RR and MVV were reduced after high pressure exposure. Due to $\mathrm{T}=60 \mathrm{~s} / \mathrm{RR}$, the duration of every breathing is prolonged after high pressure exposure (Table 7, Figure 2-E, Figure 2-F \& Figure 2-G).

Table 7: Tendency of ventilation function (MV, RR, MVV, VR \%) in EG and CG after exposure to high pressure

\begin{tabular}{|l|l|l|l|l|l|l|l|l|}
\hline & \multicolumn{2}{|l|}{ MV } & \multicolumn{2}{l|}{ RR } & \multicolumn{2}{l|}{ MVV } & VR\% \\
\hline & tendency & P-Value & tendency & P-Value & tendency & P-Value & tendency & P-Value \\
\hline EG & $\downarrow$ & $>0.05$ & $\downarrow$ & $>0.05$ & $\downarrow$ & $>0.05$ & $\uparrow$ & $>0.05$ \\
\hline CG & $\downarrow$ & $>0.05$ & $\downarrow$ & $>0.05$ & $\downarrow$ & $>0.05$ & $\uparrow$ & $>0.05$ \\
\hline
\end{tabular}

\section{Discussion}

The physiological factors affecting static lung volume are many, including age gender height, weight BMI, race posture, physical activity levels, altitude, etc. Predictions can be made for normal lung capacity based on these physiological factors, and the functional prediction equation for lung function is applicable to people between the ages of 3 and 95 [19-28]. But these lung function reference range is not covered at all, because the limits of the normal range of lung volume and capacity in different geographical, age, gender, and ethnic groups are still blurred. Although there is a high accuracy in predicting lung function, however, the measurements are not $100 \%$ identical to the measured values. The lower limit and upper limit of the acceptable range is between $80 \%$ and $120 \%$ of the predicted value [29-31]. Prediction of lung function was applied to the Baldwin equation of regression, which also suggested estimates between $80 \%$ and $120 \%$ of the ratio to actual and predicted were acceptable.

There are sublabel changes and different variations in both groups 
under different experimental settings. The unanimous changes come from matching pressure loading in experimental settings and the same duration of exposure in time. While the different variations are rooted in many factors, such as the static load on SCUBA Diving equipment, increasing breathing work caused by exercise in higher density of medium environment, low temperature and immersion effect underwater in addition to the pressure exposure.

Short-term aftereffects in ventilation after pure hyperbaric exposure of $2.2 A T A$

The pressure difference between the environment and the intrapulmonary pressure increases during the hyperbaric exposure. Therefore, the impetus of inhaling increases and the partial pressure of oxygen rise with it. Breathing is regulated by bloodoxygen content of divers. Blood oxygen levels rise in the blood when hemohyperoxia suppressing breathing. Hypoxia stimulates the respiration system to work even hard, mostly on the rise of the breathing rhythm, to compensate hypoxemia. In this experiment, the participants were observed to be bradypnea in aftereffect of pressure exposure. Thus the RR declined. The value of MV is also being observed being at a lower level than in the normal circumstances. In general, when the MV reduced to hypoxia condition, the regulation of the respiration increases the RR. But in this experiment, the increase of the RR was not accelerated, which indicated the reduction of MV might be not caused by the lack of oxygen, but the shrink of gas volume under high pressure environment.

According to Boyle Mariotte law, the volume of inhaled gas drops as the environment pressure increases exponentially after high pressure exposure. That's why the value of TV increases in aftereffect of pressure exposure in research. The change in pressure causes the gas in the airway to produce different pressure profiles, bringing to lung volume dilatation and TV spread [32]. When forced inhaling, the gas inhaled is in lower density than that already was breathed in, thus increases in IRV. But when forced exhale, different density of gases mixed and the total volume of expired gas decrease, so the ERV goes down.

The increase in $\mathrm{VC}$ was due to the increase in the volume of the integrated TV, IRV and ERV. Although ERV was observed to reduce in the study, but the increasing part in the sum of TV and IRV was greater than ERV and offset the decline in ERV. FVC increases in aftereffect of hyperbaric exposure similarly. Forced expiratory breath in the first second comes from the upper alveolar, more generally, where the density of the gas is lower, so as the expiratory resistance is lesser. Thus exhaled gas volume per unit time is correspondingly larger. That's the reason for the $\mathrm{FEV}_{1.0}$ rise and $\mathrm{FEV}_{1.0} \%$ increase at the same time in the study. Under normal circumstances, $\mathrm{FEV}_{1.0} / \mathrm{VC} \%$ should be consistent with the rise of $\mathrm{FEV}_{1.0} \%$. However, in this experiment, $\mathrm{FEV}_{1.0}$ / VC \% declined as a result, illustrated that the growth of $\mathrm{VC}$ is bigger than the FVC. The probable reasons are associated with a time limit, also means a greater work of breathing cause fatigue of respiratory muscles and impact FVC. The results of random movements of unlike density of the expiratory gases always tend to be homogeneous mixing. So the volume of expired gas in per unit time undergoing the process of pressure variation is less than in stable atmospheric environment. That is to say the expiratory flow is reduced, confirmed observations, which are the PEF, FEF $_{25}$ ${ }_{75}, \mathrm{MEF}_{75}, \mathrm{MEF}_{50}$ decline. After $75 \%$ of gas of FVC being exhaled, the expiratory movement mainly squeezes the residual gas in the bottom of alveoli. And with the evacuation of airway, the reserved gas refilling makes the gas density decreases and leads to the increases of $\mathrm{MEF}_{25}$.

Short-term aftereffects in ventilation after diving exposure of $12 \mathrm{~m}$ underwater

The VC increase and the MVV and MV of the EG are both reduced as the same as the CG did. But the FVC of EG decline is difference from the CG, as opposite to the CG. Except the FVC, in contrast to the $\mathrm{CG}$, there are parameters of the expiratory flow speed rate as the PEF, $\mathrm{FEF}_{25-75}, \mathrm{MEF}_{75}$ and $\mathrm{MEF}_{50}$ also appear to speed up after hyperbaric exposure. To understand whether the decrease in FVC is related to the increase expiratory gas flow, we adopt the Bernoulli's equation that's valid for the flow of gases [33].

$p+\frac{1}{2} \rho v^{2}+\rho g\left(z+\frac{p}{\rho g}\right)=$ Constant

Where: $\boldsymbol{p}$ is the pressure at the chosen point. $\boldsymbol{p}$ is the density of the air at all points in the airflow. $v$ is the airflow speed at a point on a streamline. $g$ is the acceleration due to gravity. $\boldsymbol{z}$ is the elevation of the point above a reference plane and in the direction opposite to the gravitational acceleration. Velocity is inversely proportional to the pressure according to the Bernoulli's equation. Exhaled air velocity $\left(\mathrm{PEF}, \mathrm{FEF}_{25-75}, \mathrm{MEF}_{75}\right.$ and $\mathrm{MEF}_{50}$ ) accelerate after hyperbaric exposure is probably related to the lower pressure than before exposure.

Applying Bernoulli equation to demonstrate the relationships among the flow velocity, the pressure difference and the bronchial tube diameter. It can be expressed as followed:

$$
\begin{aligned}
& Q\left(\mathrm{~m}^{3} / \mathrm{Sec}\right)=p(\mathrm{~Pa})+\frac{1}{2} \rho\left(\mathrm{Kg} / \mathrm{m}^{3}\right) \times v(\mathrm{~m} / \mathrm{Sec})^{2}+\rho \times g\left(\mathrm{~m}^{\mathrm{S}} / \mathrm{Sec}^{2}\right) S \times L(\mathrm{~m}) \\
& v(\mathrm{~m} / \mathrm{Sec})=\frac{Q\left(\mathrm{~m}^{3} / \mathrm{Sec}\right)}{\pi \times\left[\frac{d(\mathrm{~m})}{2}\right]^{2}} \\
& S=10.3 \times \boldsymbol{n}^{2} / \mathrm{d}(\mathrm{m})^{5.33}
\end{aligned}
$$

Where $\boldsymbol{Q}$ is the gas flow. $\boldsymbol{p}$ is the density of the air at all points in the airflow. $\boldsymbol{v}$ is the airflow speed at a point on a streamline. $\boldsymbol{g}$ is the acceleration due to gravity. $\boldsymbol{L}$ is the length of the bronchus that the gas flows through. $\boldsymbol{S}$ is frictional resistance for bronchus. $\boldsymbol{d}$ is the tube diameter of the bronchus. $\boldsymbol{d}$ is the inner wall roughness of the bronchus.

As for the gases flow, we normally ignore the gravity. And the equation is simplified as followed

$$
Q=p+\frac{1}{2} \rho v^{2}=p+\frac{1}{2} \rho \frac{Q}{\pi \cdot\left(\frac{d}{2}\right)^{2}}
$$

The Ideal Gas Law states that the gas pressure, volume, quantity of matter and temperature all together affect the state of the gas.

$$
p V=n R T
$$


Where $\boldsymbol{p}$ is the pressure of the ideal gas $(\boldsymbol{p a}) . \boldsymbol{V}$ is the volume of the ideal gas $\left(\boldsymbol{m}^{3}\right) \boldsymbol{n}$. is the volume of the gas $(\boldsymbol{m o l}) . \boldsymbol{R}$ is the ideal gas constant $\left[\boldsymbol{J} /\left(\boldsymbol{m o l} \boldsymbol{k}^{\boldsymbol{o}}\right)\right]$. For any ideal gas, $\boldsymbol{R}$ is constant value about $8.314410 .00026\left[\boldsymbol{J} /\left(\mathbf{m o l} \boldsymbol{k}^{o}\right)\right] . \boldsymbol{T}$ is the thermodynamic temperature of the ideal gas $\left(\boldsymbol{k}^{o}\right)$. And the its relationship with the Celsius temperature $\boldsymbol{T}$ is: $T\left(\boldsymbol{k}^{o}\right)=273.15+\mathbf{T}\left({ }^{\circ} \mathrm{C}\right)$.

In addition to the gas pressure and density, the inner diameter of the bronchus and absolute temperature of the gas also affects the expiratory flow. The value of FVC in EG dropped after exposure not only because the effect of environmental pressure, but also some factors involved with diving work on the ventilation. Submersion increased pressure on respiratory work and energy cost of breathing while diving immersion effect easily causes more fatigued in the breathing muscles [34]. A closed-fitting diving suit exerting pressure on chest affects the ventilation of the lungs while diving and diving suits of too thick or tight can hinder the ventilation of the lungs [35]. In addition, pressure between thoracic and alveolar alters the respiratory function. When carrying a breathing gas cylinder, it adds the hydrostatic pressure to the thoracic cage, while the hydrostatic pressure of thoracic is higher the respiratory system load aggravates the change of the lung volume at the end of expiration. When the end of the expiratory lung volume increase makes the length of respiratory muscles exceed over more than the optimum initial length and lessen the contraction force [36]. Thus respiratory muscles couldn't make or sustain sufficient strain to cope with the increasing breathing work, which driving FVC to decrease.

The reliance of pulmonary circulation on gravity decreasing triggered the redistribution of cycle during immersion [37]. The increased in pulmonary circulation, pulmonary capillary hyperemia, pulmonary artery pressure and vascular volume leads to pulmonary interstitial edema and breathing membrane elasticity decreased because of its 'thickening, ultimately the residual capacity increase and $\mathrm{VC}$ dwindle [38]. Therefore, $\mathrm{FEV}_{1.0} / \mathrm{VC} \%$ increases. Peripheral circulation vessels shrink in low temperature underwater, increase circulation redistribution and pulmonary blood volume, exacerbation pulmonary interstitial edema and eventually cause airway stenosis [39]. So that expiratory flow rate increases during the expiratory phase, and PEF, $\mathrm{FEF}_{25-75}, \mathrm{MEF}_{75}$, $\mathrm{MEF}_{50}$ and $\mathrm{MEF}_{25}$ increase.

To sum up, the aftereffect of pure pressure exposure of 2.2 ATA in lung ventilation parameters of $\mathrm{VC}, \mathrm{MV}$ and $\mathrm{MVV}$ are increasing. While as a result of $12 m$ diving exposure, underwater immersion effect and low temperature of the diving environment caused pulmonary interstitial edema and small airway stenosis, makes the FVC decline, speeded the expiratory flow rate. In addition to environmental pressure, the non-pressure factors of the environment also affect the ventilation changes in the lungs.

\section{Conclusion}

Water immersion and water temperature during diving exposure could cause and accelerate hemodynamic changes in pulmonary circulation induced pulmonary interstitial edema and led to the increase of external respiratory work. Instant effects of diving exposure in the study are quite consistent with the long-term cumulative effect of professional divers in previous research, which is FVC reduced because small airway become narrower. The results illustrate even the small depth of short-range diving exercise have definite influences on pulmonary ventilation, which mainly comes from the environmental factor but not the pressure increases. The limitation of this experiment is that we consider only the physical change of ventilation instead of taking gas exchanges within the pulmonary circulation that also can influence ventilation as well. The research suggested that sufficient rest and proper compression exercise is in need in relief interval during the occupational training or working, in order to avoid the superimposed effects of every single diving exposure immediate effect which acceleration attenuation of lung function.

\section{Acknowledgements}

Special thanks are for Zhanjiang Diving School that provides experimental area and the main experimental equipment. And also like to thank Yuandong and Qi Libin, staff diving instructors from Zhanjiang Diving School who had offered help and in the experiment.

\section{References}

1. Eichhorn L, Leyk D (2015) Diving Medicine in Clinical Practice. Dtsch Arztebl Int 112: 147-158.

2. Muradyan I (2010) Inhalation heterogeneity from subresidual volumes in elite divers. J Appl Physiol 109: 1969-1973.

3. Pougnet R (2014) Longitudinal change in professional divers' lung function: literature review. Int Marit Health 65: 223-229.

4. Konarski M (2013) Lung Function in Divers, in Neurobiology of Respiration, M. Pokorski, M. Pokorski^Editors. Springer Netherlands: Dordrecht 2013; 221-227.

5. Richard P (2013) Evolution of the ventilatory function of professional divers over 10 years. Undersea Hyperb Med 40: 339-343.

6. Bove AA (2016) Pulmonary Aspects of Exercise and Sports. Methodist Debakey Cardiovasc J 12: 93-97.

7. Moon RE (2016) Swimming-Induced Pulmonary Edema: Pathophysiology and Risk Reduction with Sildenafil. Circulation 133: 988-996.

8. Coulange M (2010) Pulmonary oedema in healthy SCUBA divers: new physiopathological pathways. Clin Physiol Funct Imaging 30: 181-186.

9. Watt SJ (1985) Effect of commercial diving on ventilatory function. Br J Ind Med 42: 59-62.

10. Skogstad M, E Thorsen, T Haldorsen (2000) Lung function over the first 3 years of a professional diving career. Occup Environ Med 57: 390-395.

11. Richard P (2013) Evolution of the ventilatory function of professional divers over 10 years. Undersea Hyperb Med 40: 339-343.

12. Konarski M (2013) Lung Function in Divers, in Neurobiology of Respiration, M. Pokorski, M. Pokorski^Editors. Springer Netherlands: Dordrecht 2013: 221-227.

13. Weaver LK (2009) Prevalence of airway obstruction in recreational SCUBA divers. Wilderness Environ Med 20: 125-128.

14. Davey IS, JE Cotes, JW Reed (1984) Relationship of ventilatory capacity to hyperbaric exposure in divers. J Appl Physiol Respir Environ Exerc Physiol 56: 1655.

15. Richard P (2013) Evolution of the ventilatory function of 
professional divers over 10 years. Undersea Hyperb Med 40: 339-343.

16. Skogstad M (2002) Lung function over six years among professional divers. Occup Environ Med 59: 629-633.

17. Moore GS (2009) Ventilation-perfusion inequality in the human lung is not increased following no-decompressionstop hyperbaric exposure. Eur J Appl Physiol 107: 545-552.

18. Tepper RS (2012) Asthma Outcomes: Pulmonary Physiology. J Allergy Clin Immunol 129: S65-S87.

19. Sharma G, J Goodwin (2006) Effect of aging on respiratory system physiology and immunology. Clin Interv Aging 1: 253-260.

20. Carey MA (2007) The impact of sex and sex hormones on lung physiology and disease: lessons from animal studies. Am J Physiol Lung Cell Mol Physiol 293: L272.

21. Hsia CC, DM Hyde, ER Weibel (2016) Lung Structure and the Intrinsic Challenges of Gas Exchange. Compr Physiol 6: 827-895.

22. Jones RL, MU Nzekwu (2006) The Effects of Body Mass Index on Lung Volumes. Chest 130: 827-833.

23. Zavorsky GS (2007) Waist-to-Hip Ratio Is Associated with Pulmonary Gas Exchange in the Morbidly Obese. Chest 131: 362-367.

24. Kamal R (2015) Alterations in Lung Functions Based on BMI and Body Fat \% Among Obese Indian Population at National Capital Region. Nepal J Epidemiol 5: 470-479.

25. Whittaker A, A Sutton, C Beardsmore (2005) Are ethnic differences in lung function explained by chest size? Arch Dis Child Fetal Neonatal Ed 90: F423-F428.

26. Nielsen KG, K Holte, H Kehlet (2003) Effects of posture on postoperative pulmonary function. Acta Anaesthesiol Scand 47: 1270-1275.

27. Zemková E, D Hamar (2014) Physiological Mechanisms of Post-Exercise Balance Impairment. Sports Med 44: 437-448.

28. Quanjer PH (2012) Multi-ethnic reference values for spirometry for the 3-95-yr age range: the global lung function 2012 equations. Eur Respir J 40: 1324-1343.

29. Miller MR (2011) Interpreting Lung Function Data Using 80\% Predicted and Fixed Thresholds Misclassifies More Than 20\% of Patients. Chest 139: 52-59.

30. Mannino DM, E Diaz (2012) Guzman, Interpreting Lung Function Data Using $80 \%$ Predicted and Fixed Thresholds Identifies Patients at Increased Risk of Mortality. Chest 141: 73-80.

31. Hansen JE (2011) Lower Limit of Normal Is Better Than 70\% or $80 \%$. Chest 139: 6-8.

32. Andersson B (2011) End-expiratory lung volume and ventilation distribution with different continuous positive airway pressure systems in volunteers. Acta Anaesthesiologica Scandinavica 55: 157-164.

33. Falahatpisheh A (2016) Simplified Bernoulli's method significantly underestimates pulmonary transvalvular pressure drop. J Magn Reson Imaging 43: 1313-1319.

34. Held HE, DR Pendergast (1985) Relative effects of submersion and increased pressure on respiratory mechanics, work, and energy cost of breathing. J Appl Physiol 114: 578.

35. Schellart NA, W Sterk (2016) Influence of the diving wetsuit on standard spirometry. Diving Hyperb Med 3: 138-141.

36. Pendergast DR, CEG Lundgren (1985) The underwater environment: cardiopulmonary, thermal, and energetic demands. J Appl Physiol 106: 276.

37. Rohdin M (1985) Effects of gravity on lung diffusing capacity and cardiac output in prone and supine humans. J Appl Physiol 95: 3 .

38. Lundgren CE (1984) Respiratory function during simulated wet dives. Undersea Biomed Res 11: 139-147.

39. Uhlig F (2014) Lung function after cold-water dives with a standard scuba regulator or full-face-mask during wintertime. Diving Hyperb Med 44: 70-73.
Copyright: (C2021 Hua CHENG. This is an open-access article distributed under the terms of the Creative Commons Attribution License, which permits unrestricted use, distribution, and reproduction in any medium, provided the original author and source are credited. 